\section{Ce que vous devriez savoir pour votre formation postgraduée}

\author{
M. Giger ${ }^{a}$, S. Stöhr ${ }^{b}$, Ch. Hänggelic
}

a membre du Comité central, président de la CFPC

b membre du Comité central, vice-présidente de l'ASMAC

c avocat, responsable du Département de la formation postgraduée et continue

Où puis-je m'adresser pour les questions de formation médicale postgraduée?

Le Département de la formation postgraduée et continue (DFPC) de la FMH est compétent pour toute question concernant la formation postgraduée et continue. Vous trouverez toutes les informations nécessaires à notre page d'accueil (www.fmh.ch $\Rightarrow$ [FP \& FC]). Nous nous tenons bien entendu à votre disposition durant les heures de bureau pour tout complément d'information (Elfenstrasse 18, Case postale 293, 3000 Berne 16, tél. 03135911 11, fax 03135911 12, e-mail: fmhdipl@hin.ch).

Veuillez en outre consulter sous la rubrique Europe/accords bilatéraux de notre page d'accueil, le "Guide FMH/OFSP", lequel vous fournira des informations précieuses en prévision de l'entrée en vigueur des accords bilatéraux avec l'Europe, notamment en ce qui concerne:

- l'obtention de diplômes d'études, de titres de formation postgraduée et de certificats de formation continue;

- la reconnaissance des diplômes étrangers et des titres de formation postgraduée;

- l'autorisation de pratiquer la médecine en relation avec la politique sanitaire, le droit des assurances sociales et le droit des ressortissants étrangers.

\section{Quelles conditions dois-je remplir pour obtenir un titre spécifique de formation postgraduée?}

Les principes généraux et fondamentaux de la formation postgraduée des médecins sont réglés par la Réglementation pour la formation postgraduée (RFP) de la FMH. De plus, il existe 44 programmes de for-

Correspondance:

Christoph Hänggeli

FMH

Elfenstrasse 18

CH-3000 Berne 16

E-mail: ch@hin.ch mation postgraduée, autrement dit, un programme par titre. Nous vous invitons à prendre le temps d'étudier le programme de formation choisi, afin de vous informer des spécificités de votre curriculum (éventuelle séquence et répartition des années de formation à accomplir, fréquentation de cours, publications, etc.). La liste des établissements de formation reconnus indique en outre dans quels établissements la formation postgraduée peut être accomplie. Les stages effectués dans des hôpitaux, des cliniques ou des cabinets médicaux ne figurant pas sur la liste en question ne sont pas reconnus! Lors de votre engagement, assurez-vous qu'il s'agit bien d'un poste de formation postgraduée pour lequel un certificat FMH est établi et veillez à recevoir un contrat de travail écrit.

\section{Puis-je aussi accomplir ma formation postgraduée à l'étranger?}

Il est fortement recommandé d'obtenir l'accord de la Commission des titres avant d'effectuer un stage à l'étranger, afin de garantir que celui-ci soit reconnu. En principe, un stage de formation effectué dans un établissement de formation étranger équivalent peut être validé en tant que formation réglementaire. Cependant, la moitié au moins de la formation postgraduée spécifique doit être accomplie en Suisse dans des établissements de formation reconnus. A ce propos, veuillez vous référer aux éventuelles dispositions particulières du programme de formation concernant la définition de la formation spécifique, la classification des établissements de formation et la validation des opérations.

\section{Puis-je accomplir ma formation postgraduée à temps partiel?}

Oui, en respectant toutefois les conditions suivantes: pour que le stage soit reconnu, le taux d'occupation ne doit pas être inférieur à 50\%. Si aucune réglementation spécifique ne figure dans le programme de formation, la disposition suivante est applicable:

La moitié au plus de la formation spécifique peut être acquise à temps partiel. La formation non spécifique peut être acquise entièrement à temps partiel.

De nombreux programmes de formation admettent d'ores et déjà que la totalité de la formation puisse être accomplie à temps partiel.

\section{Existe-t-il une durée minimale pour la reconnais- sance des périodes de formation postgraduée?}

Oui! Seules peuvent être reconnues des périodes de formation de 6 mois au moins dans un même établissement de formation (en cas d'activité à temps partiel, la durée est allongée au pro rata). A titre exceptionnel, 3 courtes périodes d'au moins 3 mois sont autorisées par spécialité (pour chaque formation approfondie, une période courte est admise). L'assistanat au cabi- 
net médical et le service militaire peuvent déjà être reconnus à partir de 1 mois d'activité ininterrompue et ne comptent pas comme période courte.

\section{Une formation volontaire est-elle reconnue?}

Une activité volontaire ne peut être validée que jusqu'à concurrence de 3 mois et compte en tous les cas comme période courte.

\section{Mon assistanat au cabinet médical ou mon activité de remplaçant est-elle reconnue?}

Une activité en qualité d'assistant ou de remplaçant au cabinet médical ne peut être validée que si le programme de formation postgraduée le prévoit. En outre, l'art. 33 de la RFP est applicable.

\section{Dois-je envoyer chaque année mes certificats de formation à la FMH?}

Non! N'envoyez vos certificats FMH que lorsque vous avez rassemblé tous les documents exigés dans le programme de formation. Chaque période de formation doit être attestée par un certificat FMH reconnu et par les éventuels formulaires d'évaluation spécifiques. Le responsable de la formation procède au moins une fois par an à un entretien d'évaluation, dont les résultats sont consignés dans les protocoles officiels.

\section{Puis-je aussi évaluer mon établissement de formation?}

La FMH effectue chaque année une enquête auprès des assistants, pour leur permettre d'évaluer en détail leur établissement de formation. Nous vous invitons à y participer activement afin de combler les éventuelles lacunes en matière de formation.

\section{Comment dois-je procéder pour ma demande de titre?}

La demande en vue de l'obtention d'un titre de spécialiste doit nous parvenir exclusivement sur une formule de demande officielle adressée à la Commission des titres. Veuillez vérifier au moyen de la liste de contrôle ci-jointe, si vous êtes bien en possession de tous les documents exigés dans le programme de formation. Veuillez nous faire parvenir des photocopies lisibles de tous les certificats FMH exigés, y compris des éventuels formulaires d'évaluation spécifiques, ainsi que des protocoles d'évaluation s'y rapportant. Les dossiers incomplets et les documents originaux vous seront retournés! Les certificats FMH ne peuvent être validés que s'ils comportent votre signature et celle du responsable de l'établissement de formation reconnu.
Liste de contrôle

de tous les documents devant être annexés à une demande de titres de spécialiste/de formation approfondie de la FMH

Veuillez envoyer uniquement des photocopies lisibles et pas de documents originaux!

$\square$ Diplôme fédéral de médecin

$\square$ Titre de docteur

$\square$ Certificats FMH

$\square$ protocoles d'évaluation

$\square$ éventuels formulaires d'évaluation complémentaire de la société de discipline médicale (p.ex. listes d'opérations)

$\square$ Attestation concernant l'examen de spécialiste

$\square$ éventuelles attestations concernant les dispositions particulières du programme de formation

$\square$ publications scientifiques

$\square$ cours de formation postgraduée et continue: radioprotection (connaissances approfondies/qualifications techniques), cours-bloc, cours de sauvetage, cours d'ultrasonographie, etc.

$\square$ participation à des assemblées annuelles/ à des congrès

Les dossiers incomplets et les documents originaux seront renvoyés à l'expéditeur à leurs frais!

\section{Quand un programme de formation est-il révisé?}

La période de validité des connaissances est très courte en médecine. Il en résulte que les exigences des divers programmes de formation changent relativement rapidement. Veuillez régulièrement contrôler si le curriculum de formation que vous avez choisi a été réactualisé (www.fmh.ch). En règle générale, les nouvelles exigences sont introduites avec un délai transitoire de 3 ans. Autrement dit, le candidat qui termine sa formation postgraduée dans les 3 ans après l'entrée en vigueur du nouveau programme peut le faire selon les dispositions de l'ancien programme de formation. Dans le cas contraire, les dispositions du nouveau programme de formation sont applicables.

\section{Dois-je réussir l'examen de spécialiste?}

Toutes les sociétés de discipline médicale font désormais passer un examen de spécialiste. La question de savoir si vous ne devez que participer à l'examen ou si la réussite de celui-ci est exigée dépend de la spécialité choisie. A ce propos, nous vous renvoyons aux 
articles publiés dans le Bulletin des médecins suisses (BMS) ou sur le site internet de la FMH. La date d'examen et les modalités d'inscription sont publiées au moins 6 mois à l'avance dans le Bulletin des médecins suisses.

\section{En tant que membre de la FMH, dois-je verser} des émoluments?

Veuillez vous référer au tarif des émoluments que vous trouverez sur le site de la FMH (www.fmh.ch). En tant que membre de la FMH vous bénéficiez des prestations gratuites suivantes:
- renseignements et conseils pour toutes les questions se rapportant à la formation postgraduée (par ex. validation de la formation accomplie à l'étranger);

- établissement d'un plan de formation postgraduée, sur la base duquel la Commission des titres évaluera votre formation et vous indiquera, le cas échéant, les exigences de formation devant encore être remplies.

Ces prestations sont payantes pour les non-membres de la FMH.

\section{Nouveaux programmes de formation et programmes révisés au $1^{\text {er }}$ janvier 2001}

Par la présente publication, le Comité central de la Fédération des médecins suisses met en vigueur, au $1^{\text {er }}$ janvier 2001, les nouveaux programmes et les programmes révisés de formation postgraduée pour les titres de spécialiste, les formations approfondies, les attestations de formation complémentaire et les certificats d'aptitude technique ci-après:

Titres de spécialiste

- Allergologie et immunologie clinique

- Anesthésiologie

- Cardiologie

- Chirurgie plastique et reconstructive

- Endocrinologie-diabétologie

- Hématologie

- Médecine du travail

- Médecine nucléaire

- Néphrologie

- Oncologie médicale

- Ophtalmologie

- Pharmacologie et toxicologie cliniques

- Pneumologie

- Radiologie

- Radio-oncologie/radiothérapie
Formations approfondies

à adjoindre au titre de spécialiste en gynécologie:

- Médecine de la reproduction et endocrinologie gynécologique

à adjoindre au titre de spécialiste en radiologie:

- Neuroradiologie diagnostique

- Neuroradiologie invasive

- Radiologie pédiatrique

Attestation de formation complémentaire

- Médecine de plongée (SSMSH)

Certificats d'aptitude technique

- Radiologie à fortes doses angiologie (SSA)

- Radiologie à fortes doses cardiologie (SSC)

- Radiologie à fortes doses gynécologie et obstétrique (SSGO)

- Radiologie à fortes doses (CMPR)

- Laboratoire (CMPR)

Tous les programmes de formation peuvent être consultés sur le site de la FMH (www.fmh.ch $\Rightarrow$ FP \& FC $\Rightarrow$ Programmes FP). Les personnes n'ayant pas accès à l'internet peuvent commander lesdits programmes auprès du Département pour la formation postgraduée et continue de la FMH (Elfenstrasse 18, Case postale 293, 3000 Berne 16, tél. 03135911 11, e-mail: fmhdipl@hin.ch). 